\title{
Novel Textile Systems for the Continuous Monitoring of Vital Signals: design and characterization *
}

\author{
Isabel G. Trindade, Frederico Martins, Rúben Dias, Cristina Oliveira, José Machado da Silva
}

\begin{abstract}
In this article we present a smart textile system for the continuous monitoring of cardiorespiratory signals, produced and integrated with an industrial embroidery unit. The design of a T-shirt system, having embedded textile sensors and interconnects and custom designed circuit for data collection and Bluetooth transmission is presented. The performance of skin-contact textile electrodes, having distinctive electrical characteristics and surface morphologies, was characterized by measurements of signal to noise ratio, under dry and moisture conditions. The influence of the electrodes size and the wear resistance were addressed. Results of an electrocardiogram acquisition with a subject wearing the T-shirt and display on a smartphone are also shown. The presented smart textile systems exhibit good performance and versatility for custom demand production.
\end{abstract}

Index Terms-capacitive sensors, embroidery electrodes, health care, smart textiles, telemedicine, wearable systems.

\section{INTRODUCTION}

Since the 2000s, smart textiles [1], [2] were considered strategic to reduce morbidity and health care costs associated to diseases of the circulatory system. Holter monitors are ambulatory devices, currently used to capture a patient electrocardiogram (EKG) for one to three days. Although Holter systems show small dimensions and are portable, they connect to $\mathrm{Ag} / \mathrm{AgCl}$ gel electrodes through long cables, which are perceived as intrusive and uncomfortable and prone to misconnections. Functional clothing for the continuous monitoring and recording of vital signals in ubiquitous and non-invasive ways have been developed using diverse approaches [3 - 6]. The Wealthy system [3] consists of elastic garments made by seamless knitting technology, integrating knitted electrodes, interconnects and piezoresistive sensors for the continuous monitoring of EKG and pulmonary ventilation signals. The electrodes and interconnects were knitted with electrically conductive yarns, consisting of stainless steel surrounded by twisted cables of viscose.

\footnotetext{
*This work is financed by the ERDF through the COMPETE Program and by National Funds through the FCT, within project SIVIC PTDC/EEI-ELC/1838/2012 (FCOMP-01-0124-FEDER-028937), and grant contract SFRH/BD/81476/2011.

I. G. Trindade and F. Martins are with FibEnTech, University of Beira Interior, Portugal (corresponding author phone: +351- 275319700; e-mail: itrindade@ubi.pt).

Rúben Dias, Cristina Oliveira, and José Machado da Silva are with INESC TEC and Faculty of Engineering, University of Porto, Portugal.
}

Hydrogel membranes placed between the knitted electrodes and the skin were necessary to improve signal to noise ratio (SNR) [7]. The LifeShirt [4] used gel electrodes and embedded textile sensors for plethysmographic respiration monitoring [8]. The VTAM system [5] consisted of a T-shirt integrating smooth, dry EKG electrodes, with leads and treatment modules incorporated into textile woven fabrics. It used a motherboard transmission module and power supply mounted on a belt that was connected to the VTAM T-shirt through a microconnector [9]. In a similar approach, Di Rienzo et al. [10] presented satisfactory preliminary clinical tests with the MagIC system, a T-shirt in lycra and cotton integrating two woven electrodes in the thorax region produced with conductive fibers.

The types of textiles electrodes investigated may be divided in three categories; knitted based and built in the clothing, exhibiting improved performance in the presence of either hydrogel or moisture [7, 11, 12]; woven based, also typically built in clothing, but whose manufacture processing has not been presented, being typically integrated in elastic clothing made by seamless knitting technology [9, 10]; textile electrodes made by embroidery stitching [13-15].

In previous work, we presented two chest band systems for the continuous monitoring of cardiorespiratory signals, produced with a digital embroidery method [16]. These bands used skin - contact textile electrodes and either a textile piezoresistive sensor or a pair of textile capacitive sensors to capture cardiac and respiratory signals, respectively. In this article, we extend the embroidery method to produce a T-shirt cardiorespiratory surveillance system, having embedded textile interconnects, textile sensors and data acquisition and transmission (DAT) unit.

In the present design, skin-contact EKG sensors are not built-in the clothing, allowing higher versatility to find the most suitable electrode for a particular user. Textile electrodes having distinctive electrical characteristics and surface morphologies, were characterized in terms of SNR and the effect of moisture investigated on several volunteers. The influence of the sensor's lateral dimensions and wear resistance were addressed.

\section{EXPERIMENTAL}

\section{A. System Design, Materials and Methods}

Figure 1 shows the T-shirt cardiac monitoring system being proposed. 


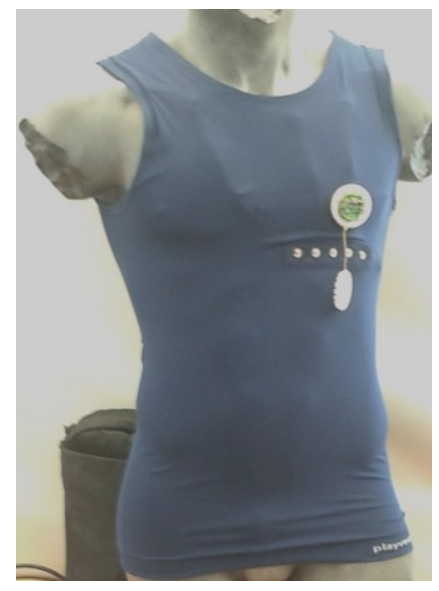

Fig. 1. The SIVIC T- shirt and data acquisition module.

This prototype integrates five snap fasteners in the configuration corresponding to the derivations of the triangle of Einthoven and Wilson leads I, II and III and the precordial V1. The T-shirt was realized by seamless knitting technology and consisted of two superimposed fabrics, integrating snap fasteners to attach the EKG electrodes. These interconnect to the DAT unit through embedded textile interconnects. The DAT unit captures the EKG signals and transmits to a smartphone or laptop through a Bluetooth link. Five snaps are also available to connect to a conventional Holter for comparison purposes.

The EKG electrodes were made by embroidery stitching [16], providing distinctive surface morphologies and electrical characteristics (see Table I), using commercial electrically conductive threads from Less EMF Inc., Imbut Gmbh and TibTech.. The electrodes are replaceable but washable and long-lasting, comfortable to wear and inexpensive.

Table I - TYPeS OF TeXTILE EleCtRodes.

\begin{tabular}{lcc}
\hline Label & $\begin{array}{c}\text { Surface } \\
\text { morphology }\end{array}$ & Resistance $(\Omega / \square)$ \\
\hline $\mathrm{A}$ & textured & 0.1 \\
$\mathrm{~S}$ & smooth & 0.2 \\
$\mathrm{D}$ & textured & 0.4 \\
$\mathrm{REF}$ & textured & $<0.1$ \\
\hline
\end{tabular}

\section{A. Characterization Method}

A 16-bit programmable data acquisition unit from National Instruments, NI-DAQ 6212, with a maximum sensitivity at \pm $0.2 \mathrm{~V}$ of $6 \mu \mathrm{V}$, was used for data acquisition. Each pair of electrodes and capacitive sensors were connected to the analog inputs of the DAQ unit in differential mode. A Labview program graphically displayed and recorded the waveforms, using two $6^{\text {th }}$-order Bessel filters; a $0.05-100 \mathrm{~Hz}$ pass-band filter, and a band-stop filter of $50 \mathrm{~Hz}$. The acquisition rate was $1 \mathrm{kHz}$. The mean and standard deviation values of SNR amplitude were extracted with a Matlab program, using 50 waveforms from each experiment. The program applied a smooth function with a span $=150$ that was subtracted to the raw data, so that the resulting waveform contained only information of the QRS complex peaks and noise amplitudes, necessary to compute the SNR amplitude,

$$
S N R^{k}=20 \log \frac{V^{s, k}}{V^{n, k}}
$$

where $V^{s, k,}$ represents the mean peak amplitude of the QRS complex, and $V^{n, k,}$ represents the mean noise amplitude along the baseline, calculated with the following expressions,

$$
\begin{aligned}
V^{s, k} & =\frac{1}{50} \sum_{i=1}^{50}\left(\left|V_{\max }^{s, k, i}-V_{\min }^{s, k, i}\right|\right) \\
V^{n, k} & =\frac{1}{50} \sum_{i=1}^{50}\left(\frac{1}{100} \sum_{j=1}^{100} 2 \cdot\left|V^{n, k, j}\right|\right)
\end{aligned}
$$

\section{B. Test structures}

Test structures with lateral dimensions of $50 \mathrm{~mm} \times 5 \mathrm{~mm}$ were made from embroidery patterns identical to those of the textile electrodes, to be used to determine their electrical characteristics and evaluate resistance to laundering cycles.

\section{III.RESULTS AND DISCUSSION}

\section{A. Electrodes characterization}

The SNR of gel and textile electrodes were measured in one volunteer, using a chest band with two electrodes located around the region of the intercostal V4 and V5. Table II summarizes the obtained experimental values.

The textile electrodes "REF" and "A", having similar electrical characteristics and surface morphologies, exhibited nearly identical SNR levels, about $2 \mathrm{~dB}$ below that of gel electrodes. The lower performances of electrodes " $S$ " and "D" suggest that the surface morphology and electrical characteristics, respectively, affect the SNR amplitude. In addition to the electrical characteristics, the surface of the electrode should be considered in the design of skin - contact electrodes $[17,18]$.

No measurable difference was obtained in the SNR amplitude of textile electrodes of type "A" having electrode

TABLE II - SNR VALUes ObTAINED For DifFERENT ELECTRODES.

\begin{tabular}{ll}
\hline Type of electrode & SNR $(\mathrm{dB})$ \\
\hline gel & $31.7 \pm 0.2$ \\
REF & $29.9 \pm 1.1$ \\
A & $30.1 \pm 0.4$ \\
D & $25.1 \pm 0.2$ \\
S & $27.8 \pm 0.5$ \\
\hline
\end{tabular}


diameters of $12 \mathrm{~mm}, 16 \mathrm{~mm}$ and $20 \mathrm{~mm}$. Nevertheless, the intercostal position could be easier to find with a specific size of electrode, which may be related to aspects of bone structure.

The SNR amplitudes of type "A" electrodes, under dry and moisture conditions and of gel electrodes, were measured on six volunteers using the chest band system connected to the NI-DAQ unit. The results are shown in Fig. 2. The lowest SNR amplitude was obtained with dry electrodes. The textile electrodes with moisture typically exhibit SNR amplitudes similar to those obtained with gel electrodes. The SNR increase from dry to moisture conditions varied between $2 \mathrm{~dB}$ (volunteers \#3 and \#4) to $6 \mathrm{~dB}$ (volunteer \#1).

The effect of sweat in the performance of the dry electrodes was addressed by monitoring the SNR as a function of time. The results obtained corroborated that, after a few minutes, the SNR amplitude increases as the electrodes go from dry to moisture condition. Similar results were obtained with a volunteer in motion, wearing the chest band connected to a Holter, showing unstable signals during the first minutes of the essays, but stabilizing afterwards, being the captured signals similar to those obtained with gel electrodes.

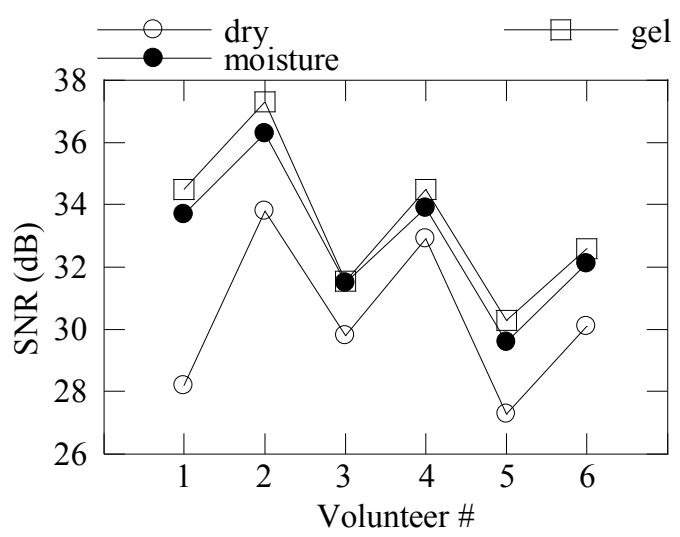

Fig. 2. SNR of gel and textile electrodes under dry and moisture conditions, obtained with various volunteers.

\section{B. Wear Resistance}

The test structures with the various types of embroidery patterns and respective electrodes were submitted to 30 washing cycles and their electrical resistance and SNR performance measured, respectively. No measurable difference was obtained in relation to those prior to washing. The high compactness of the embroidery patterns provides the electrodes with very good wear resistance.

\section{C.Electrode-skin impedance}

The electrode-skin interface is constantly changing, thus affecting the signal integrity and degrading at times the quality of the captured signal. A forearm sleeve provided with textile electrodes was used to study the electrode-skin impedance and the SNR of surface electromyographic (EMG) signals on a long-term use basis. The sleeve can be adjusted for different levels of tightening to control the pressure applied on the electrodes. The obtained results provided valuable information on the pressure that the textile garments of a sleeve or vest should apply on the recording electrodes, in order to assure a good electrical and mechanical contact between the electrodes and the skin and decrease the noise due to motion. It was observed that the electrode-skin impedance magnitude or phase is not sufficient to establish a relation with the SNR. The extraction of parameters from an electrical equivalent model of the electrode-skin interface allows determining a relation with the model parameters and the SNR. The evaluation of these parameters during long-term monitoring will allow assessing the quality of biopotential measurements in textile electrodes [19].

\section{IV.EKG DATA ACQUISITION AND TRANSMISSION UNIT}

Figure 3 shows the SIVIC 12-lead EKG DAT unit that was developed to be integrated with the T-Shirt and transmit the data to a smartphone. It is a circular board $(30 \mathrm{~mm} \varnothing)$ with an EKG analogue front-end based on the low-power Texas Instruments 24-bit ADS1298 chip and the PAN1740 Bluetooth Low Energy (BLE) module from Panasonic. It uses the internal microcontroller (32 bit ARM Cortex M0) present on the BLE module to perform all the necessary processing operations, thus saving the cost of an external microcontroller, the additional PCB area, and power consumption.

The DAT is set with a sampling frequency of $250 \mathrm{~Hz}$, which provides a good balance in terms of data accuracy and power consumption. This frequency is adequate to ensure that phase noise does not impair the estimation of the R-wave fiducial point [20]. The average DAT power consumption during operation is around $2.5 \mathrm{~mA}$. Using a $100 \mathrm{mAh}$ battery this consumption corresponds to an autonomy of about 1.5 days.

Wireless EKG monitoring systems with a high number of leads (e.g. 12-lead) are usually designed for clinical usage, being systems with a lower number of acquisition channels (e.g. 1 to 3 channels) commonly used in ambulatory cases [21, 22]. Our system was designed having in mind its use in both clinical and ambulatory scenarios and thus the number of EKG data acquisition channels is reconfigurable. Inputs not used to capture EKG signals can be used to acquire other biosignals.

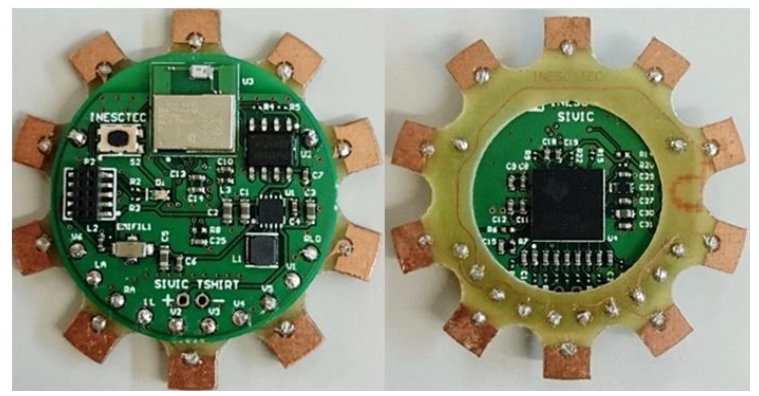

Fig. 3. The SIVIC 12-leads EKG acquisition unit prototype. 


\section{RESULTS OBTAINED WITH THE T-SHIRT}

Figure 4 shows EKG signals displayed on a smartphone, captured when a person wears the T-Shirt in a steady position and without any skin preparation. In this test, the smartphone receives three leads (LI, LII, V1) and calculates from these four more leads (LIII, aVR, aVL, aVF). The data are recorded in the smartphone but then can be sent to a PC for further analyses and features extraction.

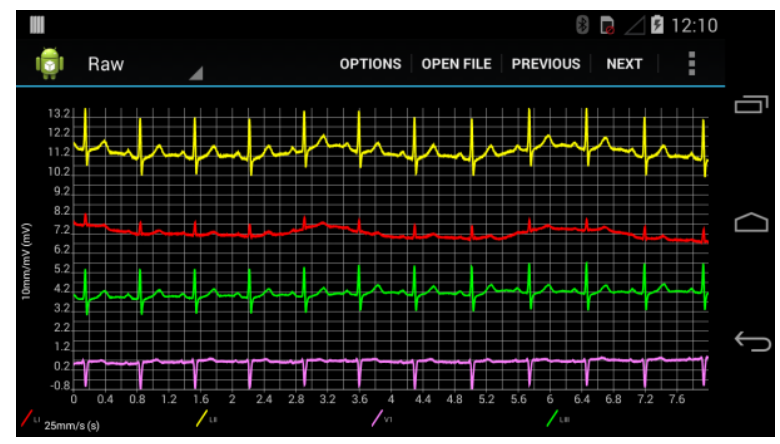

Fig. 4. Android application screenshot, representing the raw EKG signals captured when a person wears the T-Shirt.

\section{VI.CONCLUSIONS}

In this work we presented innovative smart textile systems produced by means compatible to those used in the clothing industry. The preliminary results obtained with the smart textile systems are promising in terms of versatility, performance and durability, but further tests are required, namely with volunteers showing diverse anatomic physiognomies, addressing data reliability in ambulatory conditions where motion artefacts may cause severe interference and data information lost.

In fact, wearable medical systems require high levels of dependability. These are difficult to achieve due to the intrinsic functionality of these monitoring systems, namely due to the characteristics of the electrodes being used. Data fusion models are being explored to correlate data obtained from different biosignals of the wearer, in order to obtain indicators that characterize the operation correctness of the monitoring system or a pathological condition of the wearer.

\section{ACKNOWLEDGMENTS}

This work is financed by the ERDF - European Regional Development Fund through the COMPETE Program (operational program for competitiveness) and by National Funds through FCT -Fundação para a Ciência e a Tecnologia within project SIVIC PTDC/EEI-ELC/1838/2012 (FCOMP-010124-FEDER-028937), and grant contract $\mathrm{SFRH} / \mathrm{BD} / 81476 / 2011$. The authors are also thankful to Playvest S. A. for the collaboration and manufacture of the Tshirts in lycra using seamless technology.

\section{REFERENCES}

[1] L. V. Langenhove, C. Hertleer, "Smart clothing: a new life", Int. J. Cloth. Sci. Tech. 16, pp. 63-72, 2004.

[2] M. Suh, K. Carroll and N. Cassill, "Critical Review on Smart Clothing Product Development", JTATM, 6, pp. 1-18, 2010.

[3] Wealthy System, [Online]. Available: http:// www. smartex.it/index.php/en/research/projects/european-projects / wealthy.

[4] Vivometric: LifeShirt System [Online]. Available: http://vivonoetics.com/products/sensors/lifeshirt/.

[5] VTAMN system [Online]. Available: http://george.medes.fr/ home_fr/telemedecine/assistance_aux_personnes/vtamn.html.

[6] MyHeart system [Online]. Available: http://www.research.philips.com/ technologies/myheart/index.html.

[7] R. Paradiso, G. Loriga and N. Taccini, "Knitted Bioclothes for Health Monitoring", in Proc. of EMBC., San Francisco, 2004, pp. 2165-2168.

[8] K. J. Heilman and S. W. Porges, "Accuracy of the LifeShirt (Vivometrics) in the detection of cardiac rhythms", Biol. Psychol., 75, pp. 300-305, 2007.

[9] F. Axisa, P. M. Schmitt, C. Gehin, G. Delhomme, E. McAdams, and A. Dittmar, "Flexible Technologies and Smart Clothing for Citizen Medicine, Home Healthcare, and Disease Prevention", IEEE T. Inf. Technol. B. 9 (3), pp. 325-336, 2005.

[10] M. Di Rienzo, F. Rizzo, G. Parati, G. Brambilla, M. Ferratini, P Castoglioni, "MagIC System: a New Textile-Based Wearable Device for Biological Signal Monitoring. Applicability in Daily Life and Clinical Setting", in Proc. of EMBC, Shanghai, 2005, pp. 7167-7169.

[11] S. Coyle, K. T. Lau, N. Moyna, N. O’Gorman, D. Diamond, F. Di Francesco, D. Constanzo, P. Salvo, M. G. Trivellha, D. E. De Rossi, N. Taccini, R. Paradiso, J. Porchet, A. Ridolfi, J. Luprano, C. Chuzel, T. Lanier, F. Revol-Cavalier, S. Schoumacker, V. Mourier, I. Chartier, R. Convert, H. De-Moncuit, C. Bini, C., "BIOTEX - Biosensing Textiles for Personalised Healthcare Management", IEEE T. Inf. Technol. B, Vol. 14, No.2, pp. 364 - 370, 2010.

[12] A. Catarino, H. Carvalho, M. J. Dias, T. Pereira, P. S. Girão, "Continuous Health Monitoring Using E-Textile Integrated Biosensors", in Proc. of EPE, Iasi, 2012, pp. 605 - 609.

[13] M. M. Puurtinen, S. M. Komulainen, P. Kauppinen, J. Malmivuo and J. K. Hyttinen, "Measurement of noise and impedance of dry and wet textile electrodes, and textile electrodes with hydrogel", in Proc. of EMBS, New York, 2006, pp. 6012-6015.

[14] J. Löfhede, F. Seoane and M. Thordstein, "Textile Electrodes for EEG Recording - A Pilot Study", Sensors 12, pp. 16907-16919, 2012.

[15] T. Kannaian, R. Neelaveni and G. Thilagavathi, "Design and development of embroidered textile electrodes for continuous measurement of electrocardiogram signals", J. Ind. Text., pp. 303 - 318 , 2012.

[16] I. G. Trindade, F. Martins, R. Miguel, M. Santos Silva, "Design and Integration of Wearable Devices in Textiles", Sensors \& Transducers J., Vol. 183, pp. 42-47, 2014.

[17] H. Zhang, W. Li, X. Tao, P. Xu, H. Liu, "Textile-Structured Human Body Surface Biopotencial Signal Acquisition Electrode", in Proc. CISP 11, Vol. 5, 2011, pp. 2792-2797.

[18] P. J. Xu, H. Zhang, X. M. Tao, "Textile - structured electrodes for electrocardiogram", Textile Progress, 40:4, pp. 183-213, 2008.

[19] C. C. Oliveira, J. Machado da Silva, I. G. Trindade, and F. Martins, "Characterization of the electrode-skin impedance of textile electrodes" in Proc. DCIS, Madrid, 2014, pp. 1-6.

[20] P. Kligfield, L. S. Gettes, J. J. Bailey, R. Childers, B. J. Deal, E. W. Hancock, G. v. Herpen, J. A. Kors, P. Macfarlane, D. M. Mirvis, O. Pahlm, P. Rautaharju, and G. S. Wagner, "Recommendations for the Standardization and Interpretation of the Electrocardiogram, Part I", J. Am. Coll. Cardiol., 49(10), pp. 1109-1127, 2007

[21] M. K. Delano and C. G. Sodini, "A long-term wearable electrocardiogram measurement system" in Proc. of BSN, Cambridge, MA, 2013, pp. 1- 6.

[22] N. Amour, A. Hersi, N. Alajlan, Y. Bazi, and H. AlHichri, "Implementation of a mobile health system for monitoring ECG signals," in Proc. of ASE BigData / SocialInformatics / PASSAT / BioMedCom, Cambridge, MA, 2014, pp. 1-7. 